\title{
The ATLAS experiment and its forward detector capabilities
}

\author{
María Teresa Dova ${ }^{\mathrm{a}}$, on behalf of the ATLAS Collaboration * \\ aIFLP-CONICET and Universidad Nacional de La Plata \\ Physics Department, FCE, C.C. 67, 1900 La Plata, Argentina
}

The LHC will provide pp collisions at the unprecedented center-of-mass energy of $14 \mathrm{TeV}$. The proton beams will have an energy corresponding to $0.1 \mathrm{EeV}$ in the rest frame of the target protons, approaching the region of the ultra-high energy cosmic rays where the lack of suitable accelerator data is the dominant source of systematic uncertainties in the analysis. The ATLAS experiment at the LHC is building detector systems to cover the forward region. The status of the ATLAS experiment and its forward detectors at the start-up of LHC is presented. The ATLAS forward physics program that will contribute to understand high energy hadronic interactions and help determining the primary cosmic ray composition is discussed.

\section{Introduction}

Particle colliders and ultra high energy cosmic ray (UHECR) experiments are at present the best tools to test the deep structure of matter and the interactions of its fundamental constituents at the high energy frontier.

UHECR interactions are orders of magnitude beyond what can be achieved in current terrestrial collider experiments and may open a window to energy and kinematic regions previously unexplored in the study of fundamental interactions. The Pierre Auger Collaboration, exploring the potential of the hybrid design of the observatory, has developed a method to obtain the energy spectrum which does not rely on models [1]. However, the primary cosmic ray composition in UHECR can only be determined by detailed simulation and extrapolation of the accelerator data to the highest cosmic ray energies. The Large Hadron Collider (LHC) [2], will provide $p p$ collisions at the unprecedented centre-of-mass energy of $\sqrt{s}=14 \mathrm{TeV}$ and luminosity of $\mathcal{L}=10^{34}$ $\mathrm{cm}^{-2} \mathrm{~s}^{-1}$. ATLAS [3] is one of the two general purpose detectors built to probe p-p collisions at LHC. To cover the forward region the ATLAS ex-

\footnotetext{
*We are greatly indebted to all CERNs departments and to the LHC project for their immense efforts. We are grateful to all the funding agencies which supported the construction and the commissioning of the ATLAS detector and also provided the computing infrastructure.
}

periment is building dedicated detector systems: a Cerenkov detector called LUCID [4], a Zero Degree Calorimeter [5] and Roman Pots which will house a scintillating fiber tracker system called ALFA [6]. This paper covers the status of the ATLAS detector and capabilities of the forward detectors designed to perform a variety of measurements ranging from the absolute luminosity, total $p p$ cross section and a wide forward physics program.

\section{The ATLAS Detector}

The ATLAS collaboration, consisting of about 2500 scientists from 169 institutes in 37 countries, has built the ATLAS detector, a roughly cylindershaped with a length of $44 \mathrm{~m}$ and a diameter of $25 \mathrm{~m}$. It is installed in a cavern $92 \mathrm{~m}$ below ground at CERN. A schematic layout of ATLAS is shown in Figure 1. The scale and general design is determined by the physics goals of LHC, mainly excellent resolution and hermeticity of the calorimeters, a very good muon momentum resolution and inner detector performance for heavy flavor identification.

The inner detector is immersed in a $2 \mathrm{~T}$ solenoidal field. Pattern recognition, momentum and vertex measurements and electron identification are achieved with a combination of discrete, high-resolution semiconductor pixel and strip de- 


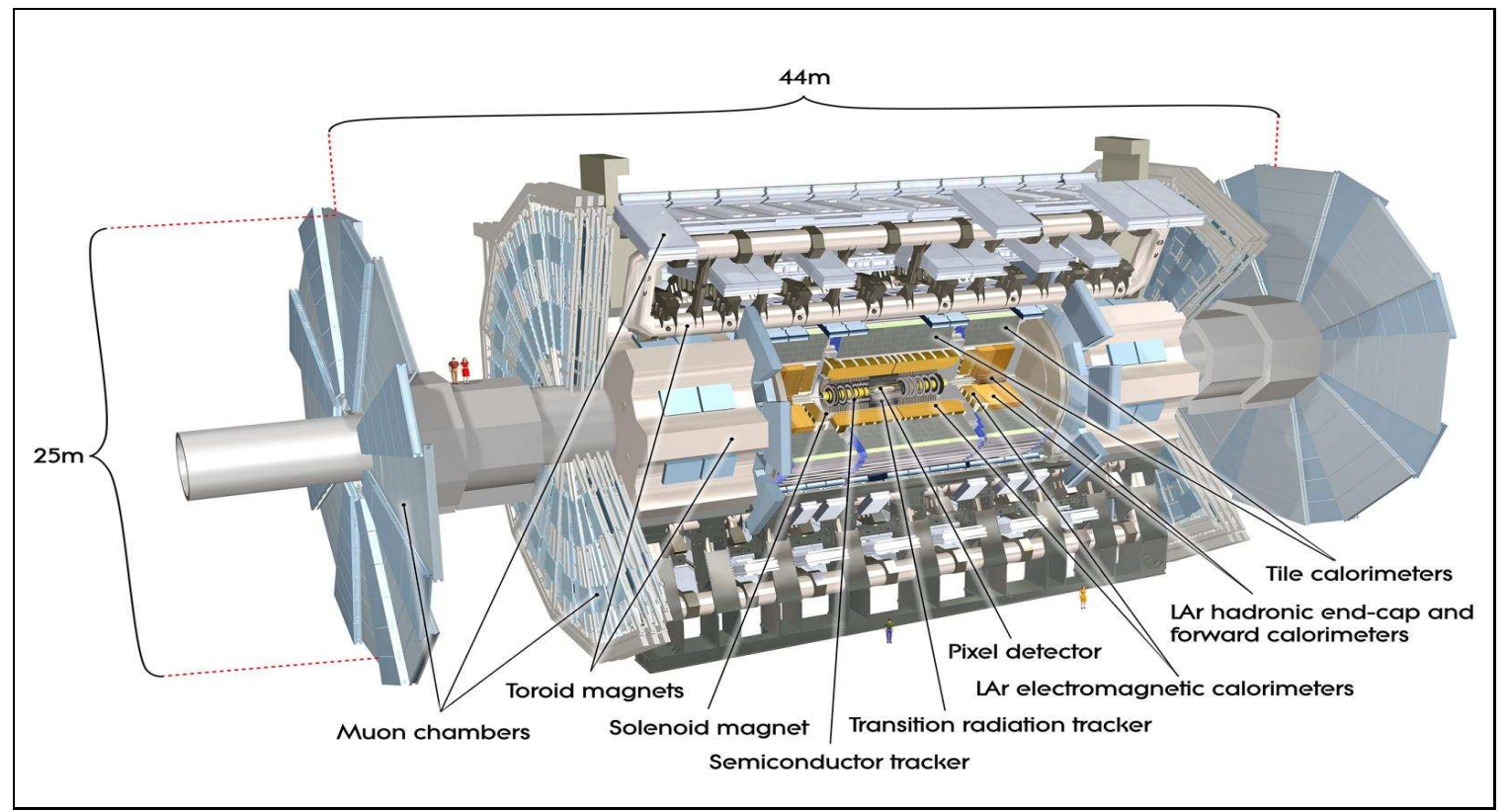

Figure 1. Layout of the ATLAS detector.

tectors in the inner part of the tracking volume, and straw-tube tracking detectors with the capability to generate and detect transition radiation in its outer part, having a total length of $5.7 \mathrm{~m}$ and a radius of $115 \mathrm{~cm} \mathrm{[3].} \mathrm{The} \mathrm{transition}$ radiation tracker (TRT) barrel, with all services fully connected and tested was ready for cosmic tests in its final position in the ATLAS detector in November 2006. The ATLAS strip detector was moved inside the ATLAS TRT in February 2007, while the installation of the ATLAS pixel detector was done in June 2007. The ID volume was sealed with its complex environmental system in April 2008.

Typically 3 pixel layers and 8 strip layers are crossed by each track. A large number of tracking points is provided by the TRT in the outer part. The ATLAS inner detector is being commissioned with cosmic rays, an example is shown in Figure 2: a cosmic ray event going through the pixel and strip detectors. Shown are the XY view and an RZ view. The track has a hit in each of the layers in both the upper and the lower hemisphere. In ATLAS combined data taking runs, several hundred millions of cosmic tracks were recorded of which about 250000 were pointing to and recorded in the pixel detector. The reconstruction, alignment, calibration and monitoring chains have been put in place to deal with real data and cosmic rays events and are providing prompt feedback to the detector performance. Results show that the detector is well within specifications in terms of noise, efficiency and resolution [7].

The calorimeter system covers the range $|\eta|<$ 4.9 using different techniques suited to cover a wide range of physics processes and to stand the radiation environment over this large $\eta$ range [3]. The Liquid Argon (LAr) Calorimeter system is composed of an electromagnetic (EM) calorimeter in the barrel and endcap regions as well as hadronic and forward calorimeters in the endcaps. The EM part of this detector is made of lead absorbers and liquid argon as active ma- 


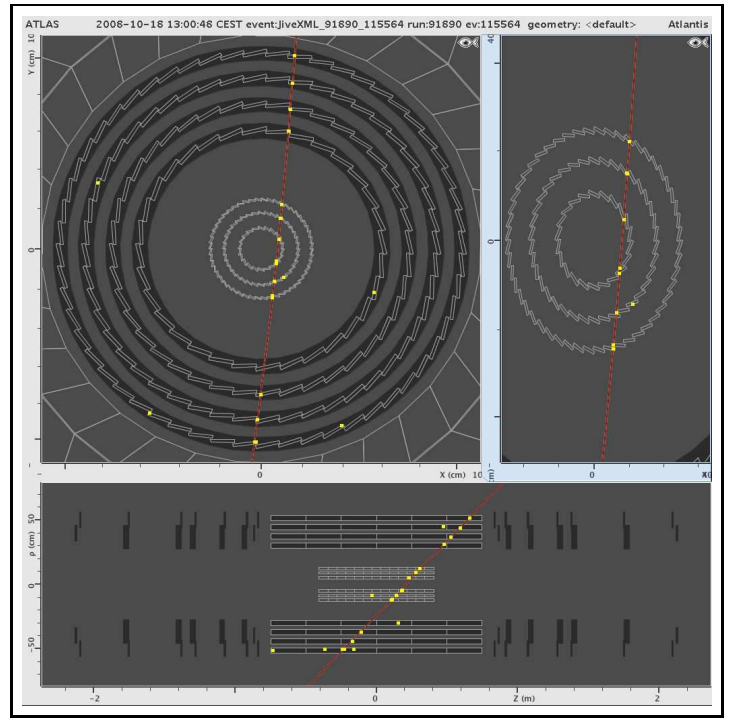

Figure 2. A cosmic ray event going through the pixel and strip detectors taken on October 18th 2008.

terial. The hadronic section uses copper absorbers. The different calorimeter parts are located in cryogenic vessels (one barrel section with EM calorimeter and two endcap sections with EM, hadronic and forward calorimeters). These vessels are surrounded by the Tile Calorimeter, the hadronic ATLAS calorimeter for lower pseudorapidity regions based on iron absorbers and scintillating plates. The forward calorimeters extend the calorimetric coverage from pseudorapidity $|\eta|>3.1$ to 4.9 and therefore sit close to the beam. The fine granularity of the EM calorimeter is ideally suited for precision measurements of $e$ and $\gamma$. The coarser granularity of the rest of the calorimeters is sufficient to satisfy the physics requirements for jet reconstruction and $E_{T}^{\text {miss }}$ measurements. In Figure 3 a view inside the liquidargon calorimeter endcap is shown as in December 2003. The calorimeter commissioning was in a mature state for the LHC start-up in September 2008, given the up to two years of operational experience since underground installation.

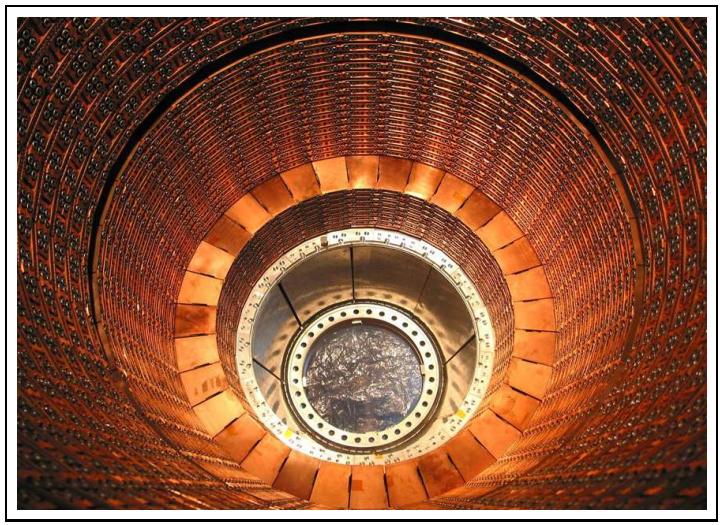

Figure 3. A view inside the liquid-argon calorimeter endcap. The circular inner bore of the EMEC, front and rear HEC wheels. December 2003

The precision measurement of muon tracks is performed, over most of the spectrometer acceptance, by Monitored Drift Tube (MDT) chambers. Three layers of MDT chambers arranged in concentric cylinders constitute the barrel MDT system. In the forward region, MDT chambers are installed in three wheels, with a coverage up to $|\eta|=2.7$. The MDT chambers are continuously monitored for misalignments and deformations by a network of optical lines, which allow for the achievement of the design resolution on sagitta measurements. In the forwardmost part of the inner layer of the muon spectrometer, where the background rate is expected to reach $1 \mathrm{kHz} / \mathrm{cm}^{2}$, Cathode Strip Chambers (CSC) are used. Muons are triggered by three double layers of Resistive Plate Chambers (RPC) in the barrel region $(|\eta|<$ 1.05), and by three stations of Thin Gap Chambers (TGC) in the endcap region $(1.05<|\eta|<$ $2.4)$. The largest part of the muon spectrometer is embedded in a toroidal magnet field, provided by three magnets: one barrel toroid, and two endcap toroids, one per side. The total bending power is 2-6 Tm in the barrel region, and 1-7 $\mathrm{Tm}$ in the endcap regions. The four technologies of muon spectrometer chambers and the system of toroidal magnets are described in Ref.[3]. 


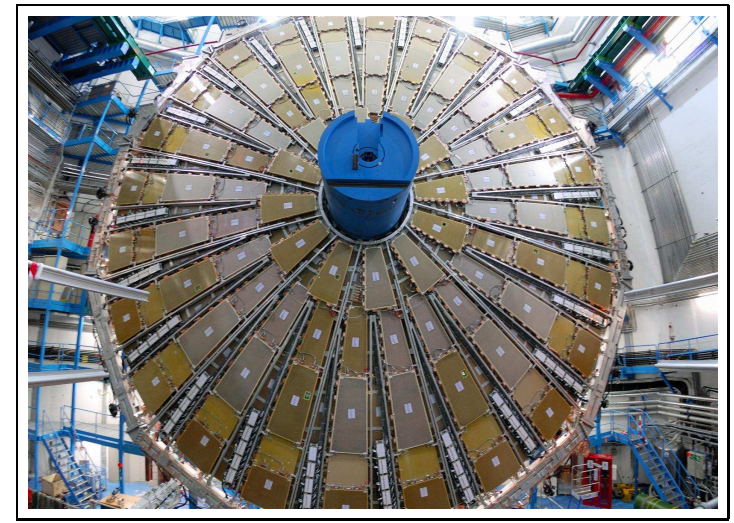

Figure 4. Installation of the first of the big wheels of the ATLAS muon spectrometer.

A picture of the installation of the first of the big wheels of the ATLAS muon spectrometer in September 2006, a TGC wheel, is shown in Figure 4. The inner stations of the muon end-cap (small wheels) were installed in March and April 2008. The outer stations were completed in June 2008, with MDT chambers and alignment bars mounted on the end walls of the ATLAS cavern. This operation was followed by the assembly of the last sections of the ATLAS beam pipe.

The full detector had been operated for a few months collecting cosmic ray data before the first single beam injection into the LHC. The experiment was kept operational continuously since end of August 2008, typically recording cosmic ray data during nights and weekends, complemented with dedicated sub-system commissioning work during week-day periods. ATLAS successfully recorded events on 10th September 2008 where the first proton beams circulating in the LHC were dumped against collimators near the ATLAS experiment. These events, named "splash" events, produced a cascade of pions and muons that were detected by the different sub-detectors in ATLAS as shown in Figure 5. After opening the collimators, the proton beams were allowed to circulate along the LHC tunnel and caused beam-gas and beam-halo events. All these events were used very efficiently for initial timing adjustments. Since the LHC incident, which occurred on 19th September 2008, the full detector was almost continuously operated until Christmas, to gather large samples of cosmic tracks for detector commissioning, calibration and alignment.

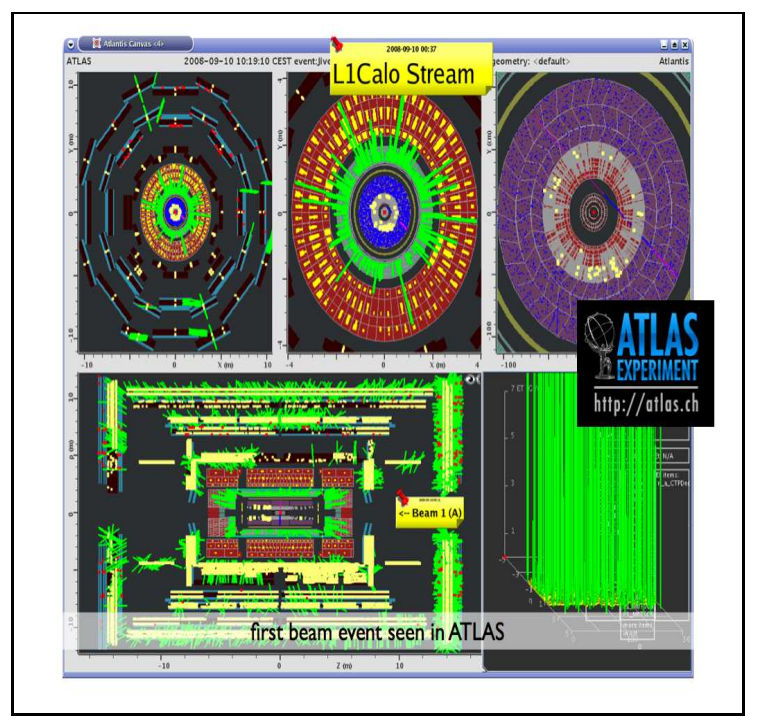

Figure 5. Picture of first collimator "splash" event seen by ATLAS. The collimator position is $140 \mathrm{~m}$ in front of the ATLAS interaction point.

ATLAS has recently completed a new set of physics studies, based on up to-date detector description and tools for reconstruction and analysis [8]. The subjects covered include the performance of trigger, ID, muon system, b-tagging, electron and photon measurement, jets and missing transverse energy, and physics studies in the areas of Standard Model, b-physics, top, Higgs, SUSY and exotics. The main focus is on physics searches based on integrated luminosities in the range of $\approx 10-100 p b^{-1}$. 


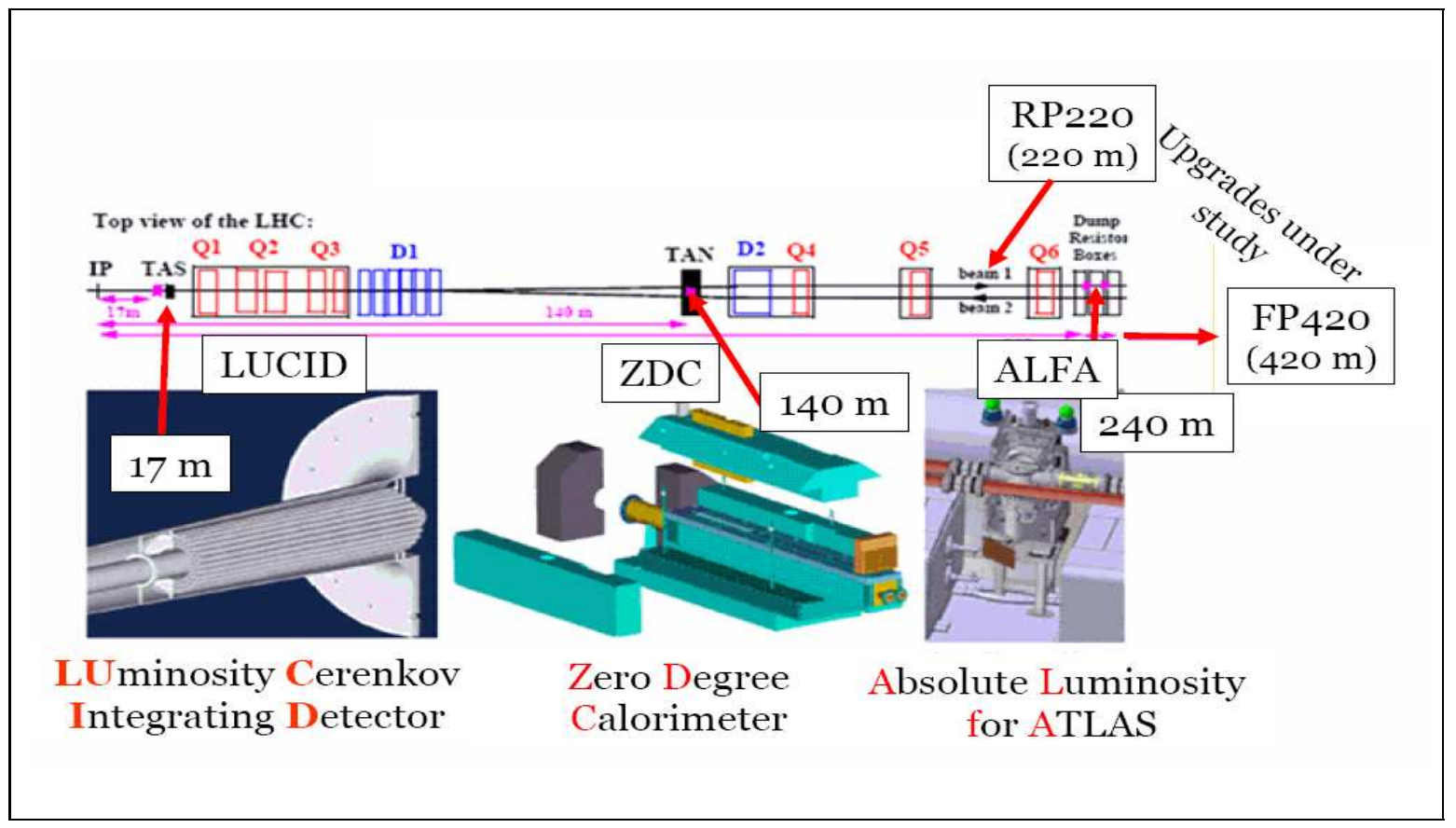

Figure 6. The ATLAS forward detectors and their position relative to the interaction point.

\section{The ATLAS Forward Detector System}

In addition to the ATLAS sub-systems mentioned above, also three dedicated systems are built to cover the forward region as shown in Figure 6 with the positions relative to the interaction point (IP). These systems are the Luminosity Cerenkov Integrating Detector called LUCID, the Zero Degree Calorimeter (ZDC) and the ALFA Roman Pot spectrometers. In the figure also the future upgrades currently under study are indicated, these are Roman Pots at 220 meters (RP220) and Forward Proton Tagging detectors at 420 meters (FP420).

The LUCID detectors are located $17 \mathrm{~m}$ from the interaction point, one on each side of ATLAS, and provide coverage of $5.6<|\eta|<6.0$ for charged particles. Each LUCID detector is a symmetric array of polished aluminum tubes that surround the beam-pipe. Each tube is $15 \mathrm{~mm}$ in diameter and filled with C4F10 gas, which gen- erates Cerenkov emission from charged particles crossing the tube. The Cerenkov light is read out by photo-multiplier tubes.

LUCID will be the only detector which is primarily dedicated to real time luminosity monitoring. LUCID is a relative luminosity detector. Its main purpose is to detect inelastic $p p$ scattering in the forward direction in order to both measure the integrated luminosity and to provide online monitoring of the instantaneous luminosity and beam conditions. Potentially LUCID could also be used for diffractive studies, for example as a rapidity-gap veto or as a tag for a diffractive signal.

For LUCID to provide the actual luminosity during a store, rather than the change in luminosity, it must be calibrated using a known absolute luminosity. Initially, this can be achieved using LHC machine parameters to an accuracy of 10-20 $\%$. In addition, Z-boson events can be used as a "standard candle" as the production cross section 
is well known from theory. This provides an absolute luminosity calibration to $5-10 \%$ accuracy. The final calibration of the absolute luminosity will be determined using elastic $p p$ scattering detected by the ALFA detectors.

The Zero Degree Calorimeter (ZDC) is located $140 \mathrm{~m}$ from the interaction point in the TAN region (target absorber for neutrals), which is the point where the single beam-pipe splits into two, and provides coverage of $|\eta|>8.3$ for neutral particles. The ZDC consists of one electromagnetic and 3 hadronic tungsten/quartz calorimeters. Vertical quartz strips provide the energy measurements and horizontal quartz rods are used for position measurement. In the first phase of LHC operation, when the number of bunches per beam is low, it is planned that the space to be occupied by the ZDC EM calorimeter, will instead be used by the LHCf experiment [9]. Later LHCf will be removed and the full ZDC installed.

The ZDC has a central role in the ATLAS heavy ion (HI) program where it is used to measure the centrality of the collisions, the luminosity as well as to provide triggers. In the HI runs the main purpose of the ZDC is to measure the spectator neutrons. These are remnants of the collision and provide information about both the magnitude and direction of the impact parameter. In addition, the ZDC has close to a $100 \%$ acceptance for $\mathrm{HI}$ collisions and together with the well known cross section of neutral particles at a zero degree angle the luminosity can be determined. In the ATLAS $p p$ program the ZDC will mainly be used to study forward particle production. The cross section measurements of particles in the forward direction at the LHC energy is of main interest to the high energy cosmic ray community which needs this information to properly model extensive air showers from UHECR. In addition the ZDC will add to the overall hermeticity of ATLAS, thus useful to suppress background in diffractive studies.

The ALFA roman pot (RP) spectrometers are located $240 \mathrm{~m}$ from the interaction point. Unlike other detectors, the RP spectrometers are not fixed relative to the beam. At injection, the ALFA detectors are in a withdrawn position far from the beam. After the beam has stabilized, the detectors are moved to within $1.5 \mathrm{~mm}$ of the beam. Elastic and diffractive protons which are not in the beam, pass through arrays of scintillating fiber trackers, which measure the distance of the proton to the beam. This is primarily to determine the absolute luminosity in ATLAS, but also other physics studies are foreseen, such as the measurement of the total $p p$ cross section, the elastic scattering parameters and, proton tagging for diffractive studies. To achieve a maximum precision in the luminosity measurement, ATLAS will measure the elastic scattering in the Coulomb interference region, which requires a measurement of scattering angles down to $\approx 3 \mu \mathrm{rad}$. To reach such small angles the LHC has to run at low luminosities with special socalled high $\beta^{*}$ optics.

At high luminosity, a diffractive physics program can be extended by installing new forward proton detectors at $220 \mathrm{~m}$ and $420 \mathrm{~m}$ on each side of the IP [10]. This opens up new possibilities to search for new physics.

\section{REFERENCES}

1. Pierre Auger Collaboration, J. Abraham et al. Phys.Rev.Lett.101:061101 (2008)

2. LHC Study Group, Conceptual Design, CERN/AC/95-05 (1995).

3. The ATLAS Collaboration, G. Aad et al. JINST 3 (2008) S08003.

4. ATLAS Forward Detectors for Luminosity Measurement and Monitoring, Letter of Intent. CERN/LHCC/2004-010 (2004).

5. Zero Degree Calorimeters for ATLAS, Letter of Intent. CERN/LHCC/2007-001 (2007).

6. ATLAS Forward Detectors for Measurement of Elastic Scattering and Luminosity, Technical Design Report. CERN/LHCC/2008-004.

7. ATLAS Collaboration (H. Hayward for the Coll.), J.Phys.Conf.Ser.119 (2008) 032023.

8. ATLAS Collaboration, Expected Performance of the ATLAS Experiment, Detector, Trigger and Physics, CERN-OPEN-2008-020.

9. A. Tricomi for the LHCf Collaboration, these proceedings.

10. M.G. Albrow et al. [FP420 R\&D Collaboration].arXiv:0706.1495 [hep-ex] 